PAPER • OPEN ACCESS

Experimental studies of differences in the distribution parameters of multidimensional spatial-subband object vectors on video images during subband information processing

To cite this article: I N Kartsan et al 2020 J. Phys.: Conf. Ser. 1679052025

View the article online for updates and enhancements.

IOP ebooks

Bringing together innovative digital publishing with leading authors from the global scientific community. Start exploring the collection-download the first chapter of every title for free. 


\title{
Experimental studies of differences in the distribution parameters of multidimensional spatial-subband object vectors on video images during subband information processing
}

\author{
I N Kartsan ${ }^{1,2}$, A O Zhukov ${ }^{3,4,5}$, B A Goloshchapova ${ }^{6}$, A N Zalivin ${ }^{6}$, I I Oleynik ${ }^{6}$ \\ and S G Orishchuk ${ }^{7}$ \\ ${ }^{1}$ Marine Hydrophysical Institute, Russian Academy of Sciences, 2, Kapitanskaya St., \\ Sevastopol, 299011, Russia \\ ${ }^{2}$ Reshetnev Siberian State University of Science and Technology, 31, Krasnoyarsky \\ Rabochy Av., Krasnoyarsk, 660037, Russia \\ ${ }^{3}$ Institute of Astronomy of the Russian Academy of Sciences, 48, Pyatnitskaya Str., \\ Moscow, 119017, Russia \\ ${ }^{4}$ FGBSI «The Federal Center of Analyzis», 33, Talalikhina St., building 4, Moscow, \\ Russia \\ ${ }^{5}$ Russian Technological University, 78, Vernadskogo Av., Moscow, 119454, Russia \\ ${ }^{6}$ Federal State Autonomous Educational Institution of Higher Education «Belgorod \\ National Research University», 85 Pobedy St., Belgorod, 308015, Russia \\ ${ }^{7}$ «Aviaavtomatika» named after V. Tarasov» Joint-Stock Company, 47, Zapolnaya str., \\ Kursk, 305040, Russia
}

E-mail: kartsan2003@mail.ru, aozhukov@mail.ru

\begin{abstract}
The variant of representation of various objects on video images in the form of samples of space-subband vectors is resulted. The technique of estimation of differences of parameters of distribution of multidimensional space-subband vectors of objects image is given. As indicators of closeness of two regions of localization of space-subband vectors of different objects, the vector of modules of difference of eigenvalues of corresponding spectral matrices of different objects, received from corresponding subband covariance matrices of objects, is used. Experimental estimations of differences in localization regions of different objects of Copter type using their video images are given. It is shown that when there is an obvious external similarity of objects, the areas of their localization differ significantly in size and orientation. It is shown that the localization region parameters of object vectors are practically invariant to object rotations on the image. The possibility of using spatially sub-band vectors and their estimates to build the decisive rules for the recognition of different objects on video images is shown.
\end{abstract}

\section{Introduction}

For successful recognition of objects in video images it is necessary to solve a number of interrelated tasks: the choice of informative features of recognition objects, the choice of a way to describe the features that minimize the computational cost of recognition, the choice of decision-making procedure in real time. Specificity of the problem of recognition of objects is a significant a priori uncertainty about 
the number of classes of objects, their features and characteristics, which does not allow the use of traditional methods of object recognition, focused on post-processing data.

There is a large number of methods and algorithms based on comparison of images, using different approaches and mathematical models. To detect and recognize repertoire marks use methods - integral, contour and characteristic points, etc. [1]. The method of characteristic points is simple to implement, has high speed, but does not provide an invariance to the shift and rotation of the object in the image. When integral methods are used, computational costs increase, because position parameters are calculated from information about the whole halftone image.

At a contour method, for recognition contours of repertoire signs are applied. In this case, recognition is conducted using the degree of similarity of the found contour and standards. This degree of similarity is determined on the basis of the correlation coefficient [2]. The implementation of this method requires the selection and analysis of a contour with high noise immunity. Here the volume of computational expenses though decreasing in relation to integral methods remains rather high [3].

There is a number of well-proven algorithms of detection of moving objects which are often used in video surveillance cameras. However, such algorithms require improvement in terms of recognition of objects and do not detect fixed objects. For example, this manifests itself in the detection of small unmanned aircraft that may remain stationary for a long time.

Application of sub-band representation of video images allows saving both spatial and frequency structure of the object image $[4,5]$. This can give an advantage over other methods that use recognition rules with preliminary determination and use of parameters of informativity of recognition signs.

\section{Sub-bandwidth presentation of video images and formation of a sample of vectors}

The video image can be represented as pixels with bit representation (e.g. $\mathrm{P}=2 \mathrm{~s}, \mathrm{~s}$ - bits) of brightness levels. The number of bits determines the number of gradations of brightness levels from black to white.

One line of the image (formed by pixels of the selected fragment of the image) can be represented as a vector [6].

$$
\vec{S}=\left(S_{1}, S_{2}, \cdots S_{Q},\right), q=1, \ldots, Q
$$

where: Q - number of pixels in a line; $\mathrm{q}$ - pixel number; $\mathrm{s}$ - number of pixel gradation levels (brightness).

In a general view, the image column formed by pixels can be similarly represented in the vector form. In the future, we will consider line views.

Change of pixel brightness in the image flow (signal components) will correspond to some "spatial frequencies".

In general, the energies of the signal components are concentrated in a small number of rather narrow intervals of the spectrum definition area. With this approach it is possible to divide the frequency axis into a number of frequency intervals [5].

$$
\Delta \omega=4 \pi /(Q-1)
$$

According to the ratio (2) it is possible to split the frequency axis into frequency intervals (sub-bands) as follows [5]

$$
\begin{gathered}
\Omega_{k}=\left[-\Omega_{1 k},-\Omega_{2 k}\right] \cup\left[\Omega_{1 k}, \Omega_{2 k}\right], \cdots k=0, \ldots, K \\
\Omega_{10}=0 ; \Omega_{20}=\frac{2 \pi}{(Q-1)} ; \Omega_{1 k}=\Omega_{2 k-1} ; \Omega_{2 k}-\Omega_{1 k}=\frac{4 \pi}{(Q-1)}
\end{gathered}
$$

where: $\mathrm{K}$ - number of frequency intervals; $\mathrm{k}$ - number of frequency interval.

The sub-band analysis is based on the concept of a part of signal energy falling within a given frequency range [5].

$$
P_{k}(S)=\int_{\omega \in \Omega_{k}} \frac{|S(\omega)|^{2} d \omega}{2 \pi}
$$

The representation of the signal directly in the original region can be written as a quadratic form. 


$$
U_{k}(\vec{S})=\vec{S}^{T} \boldsymbol{A}_{k} \vec{S}
$$

where: $\mathbf{A}_{\mathrm{k}}$ - subband matrix with elements

$$
\begin{gathered}
a_{\gamma \xi}^{k}=\frac{\sin \left[\Omega_{2 k}(\gamma-\xi)\right]-\sin \left[\Omega_{1 k}(\gamma-\xi)\right]}{\pi(\gamma-\xi)} \text { при } \gamma \neq \xi ; \\
a_{\gamma \xi}^{k}=\frac{\Omega_{2 k}-\Omega_{1 k}}{\pi} \text { при } \gamma=\xi ; \quad \gamma, \xi=1, \ldots, Q .
\end{gathered}
$$

The sub-band matrix is calculated for each frequency range $k$. After conversion (6) the vector (1) is actually converted to a vector with dimension $k$, which can be written as $[5,6]$.

$$
\overrightarrow{\mathbf{U}}=\left(\begin{array}{c}
U_{(1)} \\
U_{(2)} \\
\ldots \\
U_{(k)}
\end{array}\right)=\left(\begin{array}{llll}
U_{(1)} & U_{(2)} & \ldots & U_{(k)}
\end{array}\right)^{T}
$$

where: $U$ - fraction (part) of the signal energy in the frequency range; $k=1, \ldots, K$ - frequency range number; $T$ - transposition sign.

The vector of the species (8) can be called a space-subband vector (SSB).

The image with dimension $N$ per $Q$ pixels can be represented as a sample of $N$ volume vectors with dimension $K$.

$$
\mathbf{U}^{(N)}=\left(\begin{array}{cccc}
U_{(1) 1} & U_{(1) 2} & \cdots & U_{(1) N} \\
U_{(2) 1} & U_{(2) 2} & \cdots & U_{(2) N} \\
\cdots & \cdots & \cdots & \cdots \\
U_{(k) 1} & U_{(k) 2} & \cdots & U_{(k) N}
\end{array}\right)
$$

where: $N$ - the sample size; the first character (in brackets) for $U$-indicates the number of the frequency range; the second character for $U$ (without brackets) - indicates the number of the vector in the sample $i=1, \ldots, N$.

In this case, $N$ will indicate the number of rows in the image, and $K$ the number of frequency intervals (sub-bands).

\section{Methodology for estimation of differences in the distribution parameters of multidimensional space-sub-band vectors of object images}

In general, the SSB is a multidimensional random value. Moreover, its dimension is equal to the number of frequency intervals (sub-bands). This is determined by the fact that the process of image formation is subject to random perturbations, the probability nature of which affects all stages. This assumption allows using a statistical approach to obtaining estimates of SSB sample distribution. Probabilistic distribution of the sample can be characterized by two moments - the first initial (mathematical expectation) and the second central (covariance matrix) [7 11]. Estimation of the sample mathematical expectation vector (ME) (8) is determined by expression [7].

$$
\overrightarrow{\mathbf{m}}=\frac{1}{N} \sum_{i=1}^{N} \overrightarrow{\mathbf{U}_{\imath}}
$$

The vector $\overrightarrow{\mathbf{m}}$ size is $K$. The covariance matrix estimation is calculated in accordance with the expression [7].

$$
\mathbf{M}=\frac{1}{N-1} \sum_{i=1}^{N}\left(\overrightarrow{\mathbf{U}_{l}}-\overrightarrow{\mathbf{m}}\right)\left(\overrightarrow{\mathbf{U}_{l}}-\overrightarrow{\mathbf{m}}\right)^{T}
$$

Elements of the covariance matrix $\mathbf{M}$ reflect the degree of statistical connection of elements of the source vector of fixed parameters with each other. In fact, it is a sub-band covariance matrix (SCM) with dimensions $K \times K$.

Using SCM, it is possible to determine the area of source space, inside which a random vector is localized $\overrightarrow{\mathbf{U}}$. Such an area can be called a localization area (LA). 
Consider the geometric interpretation of LA [8]. At $\operatorname{det} \mathbf{M} \neq 0$, you can write an expression for $\mathrm{r}-\mathrm{a}$ measured ellipsoid of constant probability density $\mathrm{P}(\overrightarrow{\mathbf{U}})[8]$.

$$
\left(\overrightarrow{\mathbf{U}_{l}}-\overrightarrow{\mathbf{m}}\right)^{T} \mathbf{M}\left(\overrightarrow{\mathbf{U}_{l}}-\overrightarrow{\mathbf{m}}\right)=d^{2}
$$

for any $d>0$. The center of this ellipsoid is determined by vector components $\overrightarrow{\mathbf{m}}$. The spread relative to the center is determined by SCM M elements.

Such an ellipsoid can serve as an approximate LA model of the vector $\overrightarrow{\mathbf{U}}$ [8]. The physical sense of LA is that its dimensionality and form reflect the degree of statistical connection of the random vector elements $\overrightarrow{\mathbf{U}}$ among themselves, i.e. their pairwise correlation. Distinctions of statistical properties of (or images of objects), and hence the objects themselves, differing in size, shape and intensity of brightness, with such geometric interpretation of the concept of LA acquire clarity and concrete content [9].

As indicators of the proximity of the two regions of localization of SSB of different objects, can be used vector $\Delta \vec{l}$ modules of differences of eigenvalues $l_{k}, k=1, \ldots, \mathrm{K}$, corresponding to the spectral matrices $\boldsymbol{\Lambda}_{1}$ of object 1 and $\boldsymbol{\Lambda}_{2}$ object 2 [9].

$$
\Delta \vec{l}=\left[\begin{array}{c}
\left|\Delta l_{1}\right| \\
\left|\Delta l_{2}\right| \\
\cdots \\
\left|\Delta l_{k}\right|
\end{array}\right]=\left[\begin{array}{c}
\left|l_{1}^{1}-l_{1}^{2}\right| \\
\left|l_{2}^{1}-l_{2}^{2}\right| \\
\ldots \\
\left|l_{k}^{1}-l_{k}^{2}\right|
\end{array}\right]
$$

where: the upper index at $l$ indicates the object number, the lower index indicates the number of the spectral matrix eigenvalue corresponding to the frequency range number.

Eigenvalues of the SCM M can be found by solving the characteristic equation, which is written in the form [10].

$$
|\mathbf{M}-l \mathbf{I}|=0
$$

where: I - a single matrix.

4. Experimental study of the differences in parameters of the regions of localization of spacesubband vectors of the object image

Using the above method, experimental studies were carried out to assess differences in LA parameters of various small unmanned aerial vehicles (UAVs) of the copter type on video images.

Figures 1 and 2 show images of objects used for experimental studies.

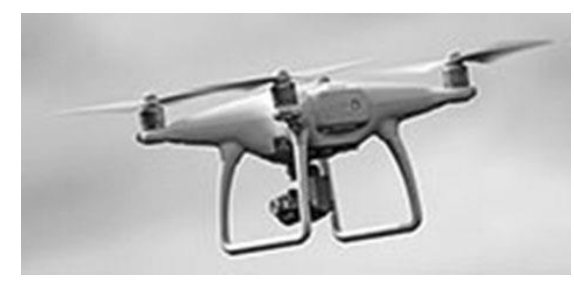

Figure 1. Object 1 (copter 1).

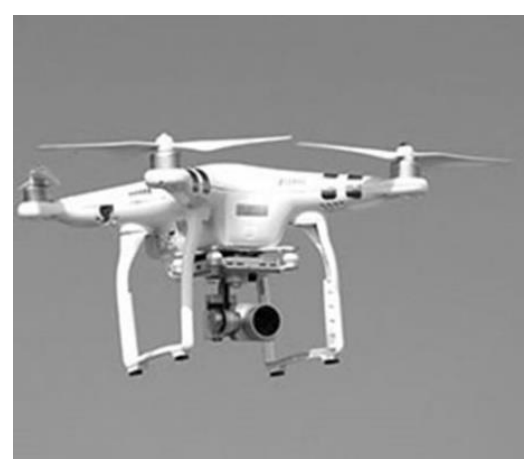

Figure 2. Object 2 (copter 2).

Figures 3 and 4 show the values of vector sizes $\overrightarrow{\mathbf{U}}$ in logarithmic scale (dB) calculated for copter 1 and copter 2 images, respectively. The number of frequency intervals was selected as $K=12$. 


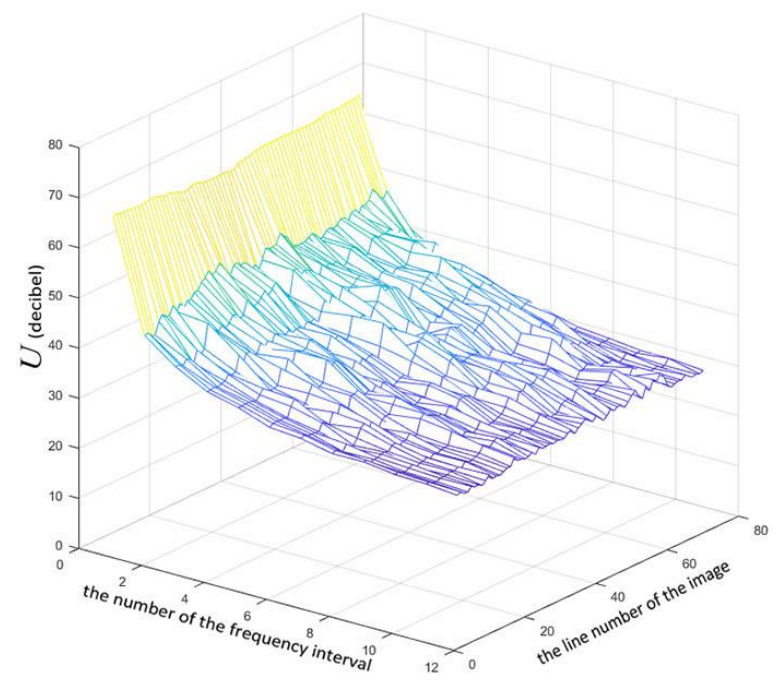

Figure 3. Values of $\overrightarrow{\mathbf{U}}$ copter vectors 1 .

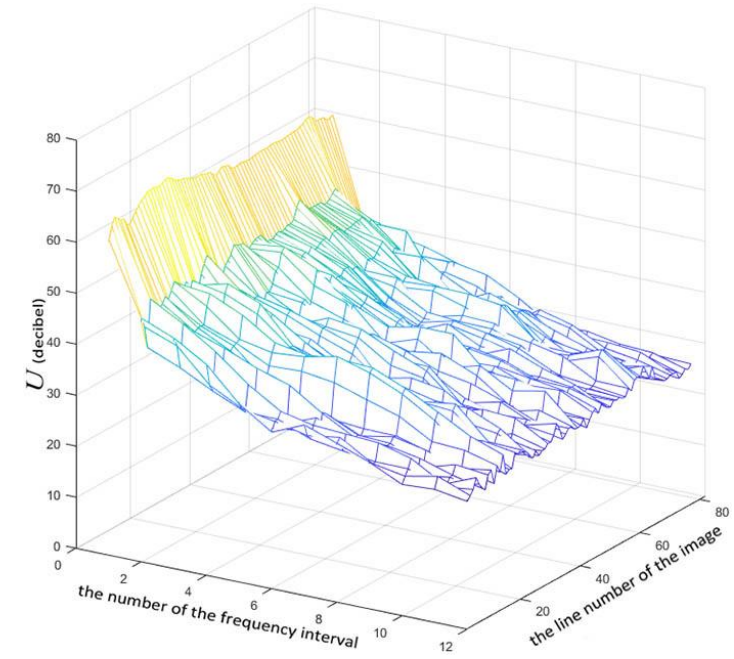

Figure 4. Values of $\overrightarrow{\mathbf{U}}$ copter vectors 2.

Having compared these drawings, it is possible to notice that the spatial-frequency structure of images of these objects has differences. It is possible to estimate numerical values of distinctions, having received estimations of a vector LA (10), SCM (11) and a vector $\Delta \vec{l}$ of modules of distinctions of own values (12).

Figure 5 shows the numerical values of the vector components $\overrightarrow{\mathbf{m}}$ (on a logarithmic scale) for images of copter 1 (blue) and copter 2 (red).

Figure 6 shows the numeric values of vector components of eigenvalues $l_{k}$ (in logarithmic scale) at $K=12$ for images of copter 1 (blue color) and copter 2 (red color).

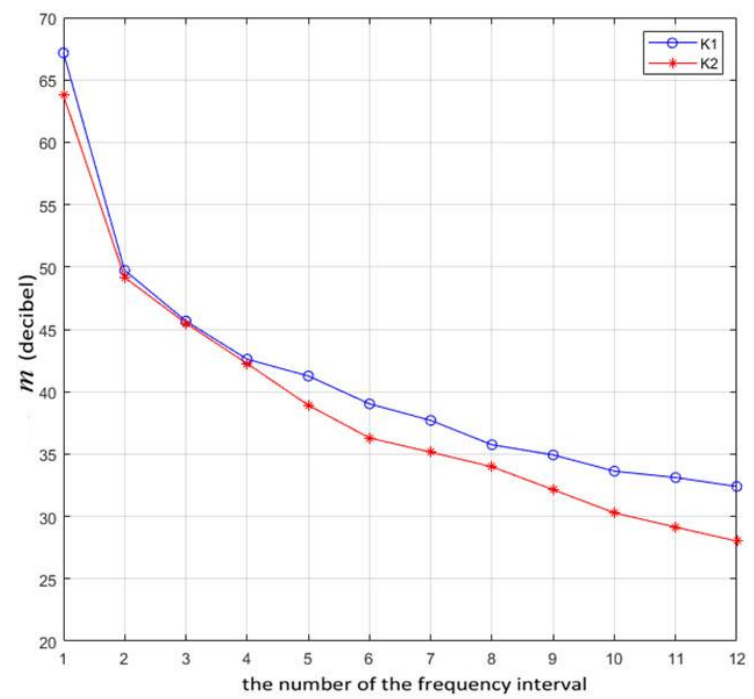

Figure 5. Values of the $\overrightarrow{\mathbf{m}}$ copter 1 and copter 2 components.

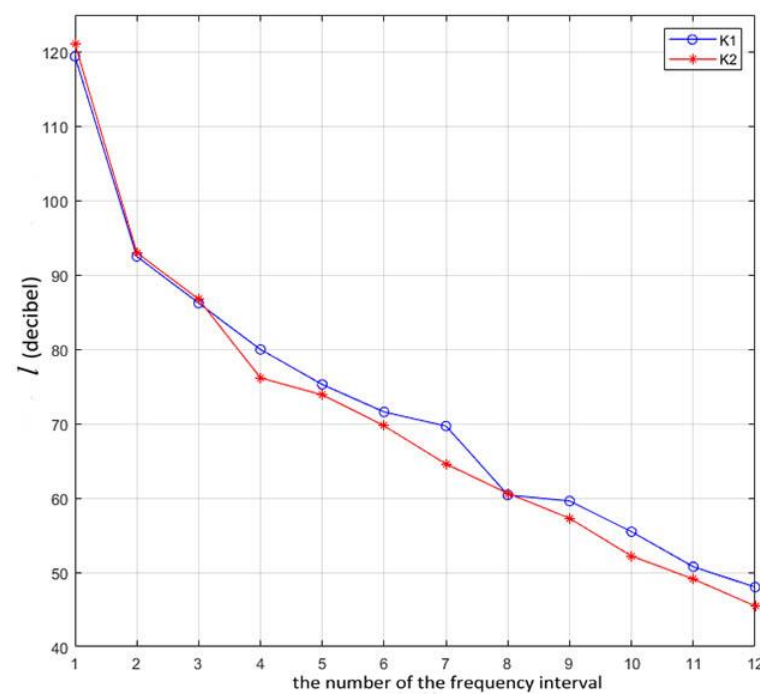

Figure 6. Values of the $l_{k}$ copter 1 and copter 2 components.

Figure 7 shows the numerical values of vector components $\Delta \vec{l}$ for two objects (in logarithmic scale).

Having analyzed the numerical values of the difference LA of copter 1 and copter 2 images, we can conclude that with the explicit external similarity of objects, their localization areas are significantly different in size and orientation. This suggests the possibility of developing decisive rules for the recognition of objects in video images using the evaluation of moments of distribution of SSB. 


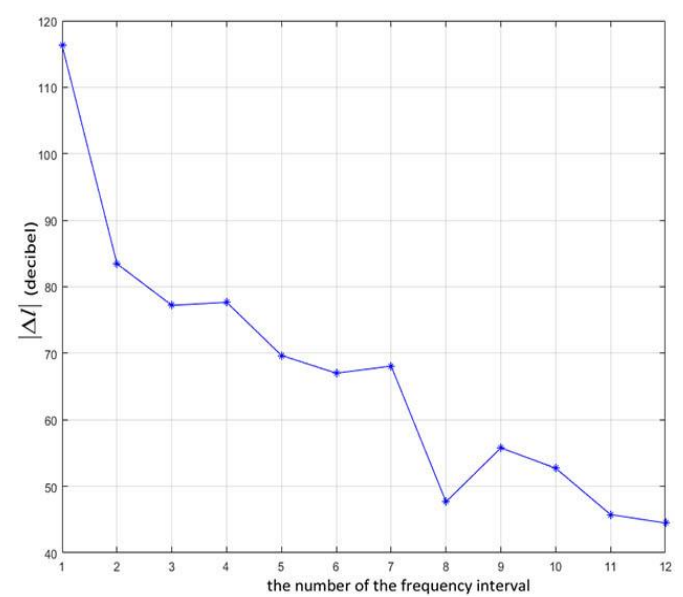

Figure 7. Value of the component of the $\Delta \vec{l}$ vector.

Besides, researches of LA parameters of one and the same object depending on spatial position, i.e. invariance to object turns on the image, are of great importance.

These experimental studies will be carried out using the method described above.

Figures 8 and 9 show the images of the object from different angles, used for experimental studies. Figure 8 shows the angle of 1, figure 9 shows the angle of 2 .

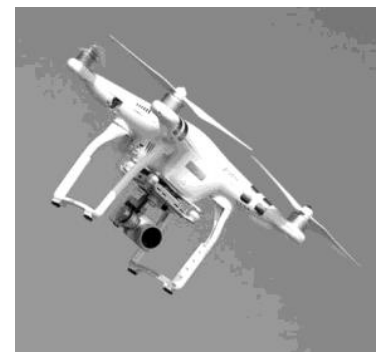

Figure 8. Angle 1.

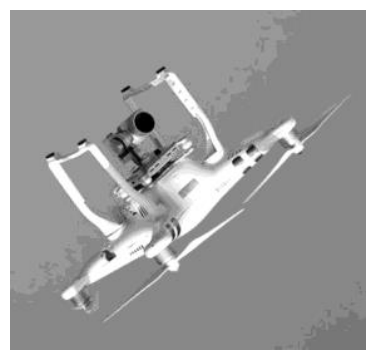

Figure 9. Angle 2.

Figure 10 shows the numerical values of vector components $\overrightarrow{\mathbf{m}}$ (in logarithmic scale), for copter images, angle 1 (blue) and angle 2 (red).

Figure 11 shows the numerical values of vector components of eigenvalues $l_{k}$ (in logarithmic scale) at $K=12$ for images of the image copter in angle 1 (blue color) and angle 2 (red color).

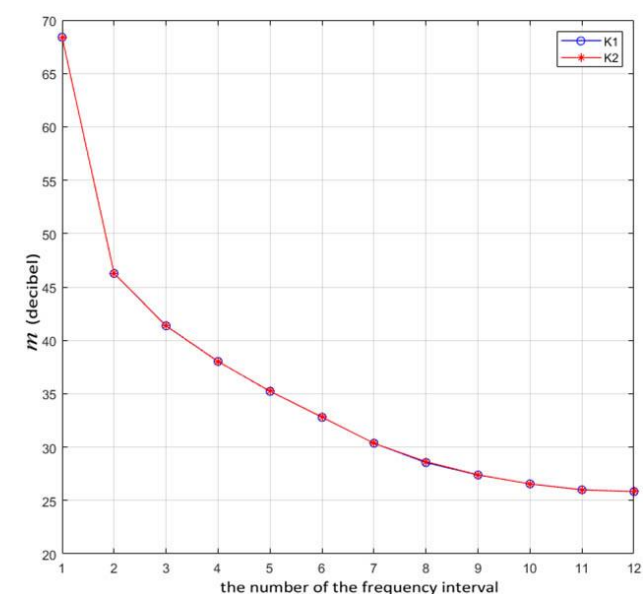

Figure 10. Values of vector components $\overrightarrow{\mathbf{m}}$ angles 1 and 2 .

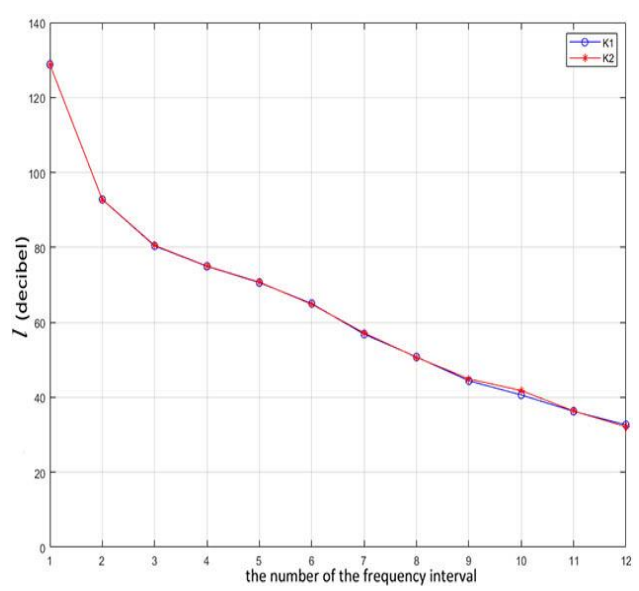

Figure 11. Values of vector components $l_{k}$ angles 1 and 2 .

Figure 12 shows the numerical values of vector components $\Delta \vec{l}$ (in logarithmic scale). 


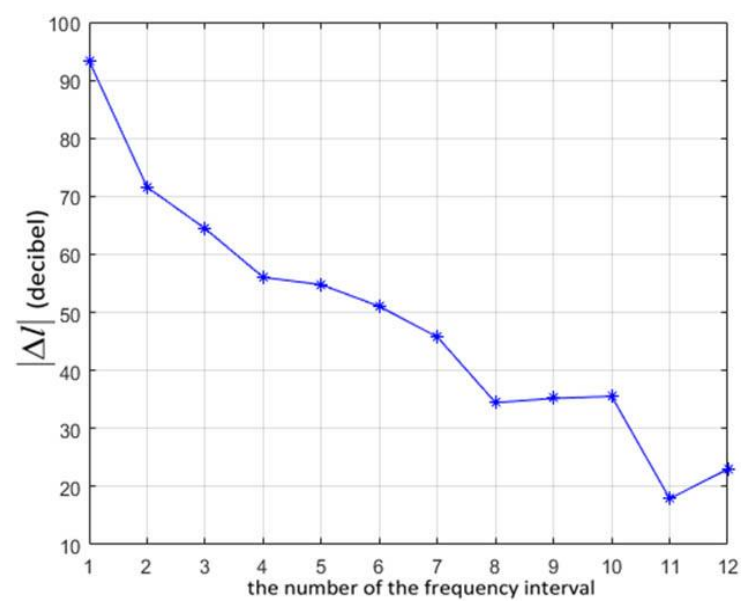

Figure 12. Value of vector components $\Delta \vec{l}$ for copter image at different angles.

The analysis of figures 10 - 12 shows that estimates of LA parameters do not have significant differences. The graphs in figures 10 and 11 practically coincide. Numerical values of vector components $\Delta \vec{l}$ are several orders less than at comparison of two different objects. It allows to conclude that LA parameters of an object are invariant to its rotation in the image. Such property of LA parameters gives preconditions for creating decisive rules for recognition of different objects. In this case, the observation angle of the object will not have a significant impact on the recognition quality.

\section{Conclusion}

This article shows the possibility of representing different objects in video images as samples of spacesub-band vectors based on sub-band analysis and signal synthesis methods.

Assuming that the SSB is a multidimensional random value, the main moments of distribution are determined - the vector of mathematical expectation and the sub-band covariance matrix. And components of the vector of mathematical expectation determine the center of an ellipsoid (or areas of localization of SSB). Spread relative to the center is determined by SCM elements.

Methodology for estimating differences in distribution parameters of multidimensional space-subband vectors of object image is developed. As indicators of closeness of two regions of localization of SSB of various objects, the vector of modules of differences of eigenvalues of corresponding spectral matrices of various objects, received from corresponding SCM objects, is used.

Experimental researches on estimation of differences in LA of different objects of copter type, using their video images, have been conducted. It is shown, that at obvious external similarity of objects, areas of their localization essentially differ in sizes and orientation. It supposes the possibility of development of the decisive rules of recognition of objects on video images with the use of estimates of moments of distribution of SSB.

Experimental researches of LA parameters of one and the same object depending on spatial position (turn) are carried out. The analysis of LA parameters allowed to conclude that they are practically invariant to the object turns on the image.

Carried out researches allow to draw a conclusion, that with the use of SSB and their estimations it is possible to construct the decisive rules of recognition of different objects on video images.

\section{Acknowledgements}

This study was supported by the Russian Federation State Task № 0827-2019-0004.

\section{References}

[1] Furman J A 2003 Introduction to Contour Analysis and its Application to Image and Signal Processing (Moscow: PHYZMATLIT, 2nd edition) 
[2] Girenko A V, Lyashenko V V, Mashtalir V P and Putyatin E P 1996 Methods of Correlation Object Detection (Kharkov: JSC «BusinessInform»)

[3] Wasin Yu G and Lebedev L I 2007 13th All-Russian Conference «Mathematical methods of pattern recognition» (Moscow: Russian Academy of Sciences) pp 285-8

[4] Zhilyakov E G, Belov S P, Oleinik I I, Babarinov S L and Trubitsyna D I 2020 Generalized sub band analysis and signal synthesis Bulletin of Electrical Engineering and Informatics 9(1) 7886

[5] Zhilyakov E G 2015 Optimal sub-band methods for analyzing and synthesizing signals of finite duration Automation and telemechanic 4 51-66

[6] Zhilyakov E G, Belov S P, Oleinik I I and Prokhorenko E I 2019 Regularization of Inverse Signal Recovery Problems HELIX the Scientific Explorer 9(2) 4883-9

[7] Fomin Ya A and Tarlovsky G R 1986 Statistical Theory of Pattern Recognition (Moscow: Radio and communication)

[8] Liebenson M N, Hesin A Y and Yanson B A 1975 Automation of TV Image Recognition (Moscow: Energiya)

[9] Goloschapova V A, Kalashnikov P A and Oleynik I I 2020 Science. Educatition. Practice: materials of the International University Science Forum (Canada, Toronto) pp 238-46

[10] Gantmacher F R 1967 Matrix Theory (Moscow: Nauka)

[11] Kartsan R V and Kartsan I N 2013 Reshetnevskie readings (Krasnoyarsk: SibGU) pp 211-2 